\title{
All about Astigmatism - Easy to Understand
}

\author{
Partha Haradhan Chow dhury ${ }^{1 *}$ and Brinda Haren Shah ${ }^{2}$ \\ 1M. Optom, Department of Optometry, Shree Satchandi Jankalyan Samiti Netra \\ Prasikshan Sansthan, Pauri, Affiliated to Uttarakhand State Medical Faculty, Dehradun, \\ India \\ 2M. Optom, Department of Optometry, Gujarat University, Gujarat, India
}

Short Communication

Volume 3 Special Issue 1

Received Date: August 02, 2018

Published Date: August 15, 2018

*Corresponding author: Partha Haradhan Chowdhury, M. Optom, Department of Optometry, Shree Satchandi Jankalyan Samiti Netra Prasikshan Sansthan, Pauri, Affiliated to Uttarakhand State Medical Faculty, Dehradun, India, Email: optometrypublish@gmail.com

\section{Introduction}

Astigmatism is a condition where always line image is created. When the parallel rays of light are coming from infinity brought to focus as a line image at different point when accommodation is at rest, condition is known as Astigmatism. The meaning of astigmatism is A- privative and stigma - point. When parallel rays of light are passing through the cylindrical surface then, horizontal meridian creates vertical line and vertical meridian creates horizontal line. The gap between horizontal line and vertical line is called "Focul Interval" or "Circle of Least Confusion"

When the rays are passing through the cylindrical surface, vertical meridian creates horizontal line and horizontal meridian creates vertical line. So, when the rays are passing through it, these rays are called "Sturm's Conoid"

When circle of least confusion is created, one vertical and one horizontal meridian is created, but when the distance between vertical meridian to retina and distance between horizontal meridian to retina are equal, then best visual acuity is achieved. This is called "Spherical Equivalent."

During correction of mixed Astigmatism, when one meridian is on the retina and another meridian is away from the retina it is called "Point of Greatest Contrast"

When the rays are passing through the cylindrical surface, there will be a particular point where divergences of vertical rays are equal to the convergence of the horizontal rays; it is called "Circle of least diffusion"

In cases of Astigmatism, always line image is created, not point image.

\section{Classification}

Astigmatism is classified as: Regular Astigmatism and Irregular Astigmatism.

\section{Regular Astigmatism}

Here two meridians are 90 degrees apart from each other. They are divided as Simple Regular Astigmatism, Compound Regular Astigmatism and Mixed Regular Astigmatism.

\section{Simple Regular Astigmatism}

It is subdivided into simple myopic astigmatism and simple hypermetropic astigmatism. In Simple Hypermetropic Astigmatism, one meridian is on the retina and another meridian behind the retina. Eg: $0.00 /+4.00$ Dcyl*180. In simple Myopic Astigmatism, one meridian is on the retina and one meridian in front of the retina. Eg: $0.00 /-4.00 * 180$.

\section{Compound Regular Astigmatism}

It is subdivided into compound myopic astigmatism and compound hyperopic astigmatism. In compound myopic astigmatism, two meridians are focused in front of the retina. Eg: -2.00 Ds/-2.00 Dcyl*180. In Compound Hyperopic Astigmatism, two meridians are focused behind the retina. Eg: +2.00 Dsph/+2.00 Dcyl*180. 


\section{Open Access Journal of Ophthalmology}

\section{Mixed Astigmatism}

Here, one meridian is focused in front of the retina and another meridian is focused behind the retina. Eg: +2.00 Dsph/-6.00 Dcyl*180.

**Here, cylindrical value is always high compared to spherical value and opposite sign.**

Regular Atigmatism can also be subdivided as:

a. With the Rule Astigmatism

b. Against the Rule Astigmatism

c. Oblique Astigmatism

\section{a. with the Rule Astigmatism}

This occurs due to upper eyelid pressure and cornea becomes steeper. So, axis will be 180+/- 30

b. AGAINST THE RULE ASTIGMATISM

Here, axis will be $90+/-30$

\section{c. Oblique Astigmatism}

Here, always one meridian will be 45 degree and another will be 135 degree. i.e. 90 degrees apart.
**Regular Astigmatism can always be Corrected Properly with Spectacles.**

\section{Irregualar Astigmatism}

Here, no rules are followed regarding axis in the presence of irregular astigmatism. It mainly occurs due to Variation of Refractive Index in Crystalline lens Trauma Keratoconus. In most cases, it is corrected by Rigid Gas Permeable Contact Lenses. Keratoplasty can be performed, but it will be dependent on corneal thickness.

\section{References}

1. William J Benjamin (2006) Borish's Clinical Refraction. 2nd (Edn.).

2. Theodore Grosvenor, Theodore P Grosvenor (2007) Primary Care Optometry $5^{\text {th }}$ (Edn.).

3. Sir Stewart Duke-Elder, David Abrams (1978) DukeElder's Practice of refraction

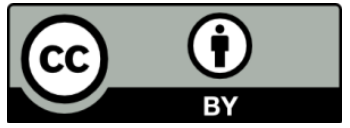

